\title{
FORMA URBANA Y MOVILIDAD SUSTENTABLE: REFLEXIONES SOBRE MONTEVIDEO.
}

Valentina Vincent 


\section{VALENTINA VINCENT}

Master in Science in Sustainable Urbanism, The Bartlett School of Planning, University College of London (Reino Unido). Arquitecta, Universidad ORT Uruguay. Profesora adjunta en Teoría y Práctica del Urbanismo, Universidad ORT Uruguay. Actividad profesional independiente.

FECHA DE RECEPCIÓN: 6 junio de 2018.

FECHA DE ACEPTACIÓN: 3 de agosto de 2018.

REGISTRO BIBLIOGRÁFICO: VINCENT, V. (2017) Forma urbana y movilidad sustentable, reflexiones sobre Montevideo. Anales de Investigación en Arquitectura, 7, 99-119. 


\section{RESUMEN}

El siguiente artículo plantea reflexiones sobre la relación entre las características de la forma física de la ciudad y la movilidad. La movilidad sustentable (aquella que prioriza los modos activos y el transporte colectivo) tiene un rol prioritario en el futuro de la planificación urbana. En la literatura, se ha estudiado ampliamente la relación entre la forma urbana y la movilidad. Varios autores plantean cuáles son las variables de la forma urbana que impactan en la movilidad, y qué se puede hacer a través del diseño para fomentar una movilidad sustentable. También se ha evaluado la importancia de tener en cuenta los factores actitudinales y cómo, si es posible, redireccionar los comportamientos de los ciudadanos hacia tendencias sustentables. Por medio del caso de Montevideo, se ejemplificará la relación de las características físicas de la ciudad con la movilidad. A su vez, se analizarán los objetivos y estrategias que plantean los planes desarrollados por el gobierno municipal con respecto al desarrollo urbano y movilidad, y se realizarán reflexiones en base a la literatura planteada.

Palabras Clave: Movilidad sustentable - Forma urbana - Planificación urbana en Montevideo.

\section{ABSTRACT}

The following article reflects on the relationship between the built environment and mobility. Sustainable mobility, which prioritizes public transit and actives modes, has a starring role in the future of urban planning. In the literature, this relationship between urban form and mobility has been vastly studied. Several authors have established which variables of urban form affect mobility, and what can be done through design to foster sustainable mobility. Also, the relevance of attitudinal factors has been studied and how, if possible, citizens' behavior could be affected in order to promote sustainable attitudes. Through the case of Montevideo, the relation between urban form and mobility will be exemplified. Also, the objectives and strategies of the city government's plans will be analyzed and conclusions will be made according to the cited literature.

Keywords: Sustainable mobility - Urban form - Urban Planning in Montevideo. 
La mayoría de las ciudades en los países en desarrollo comparten ciertas características en cuanto su movilidad: infraestructura de transporte inadecuada y deteriorada, y poca infraestructura para modos no motorizados (ciclistas, peatones). Esto ha generado que en muchas ciudades haya habido un aumento de la marginalización de los sectores más vulnerables de la población, que en general dependen de sistemas de transporte público (UN, 2013).

La movilidad sustentable (aquella que prioriza los modos activos y el transporte colectivo) tiene un rol prioritario en el futuro de las ciudades sustentables (Banister, 2008). A pesar de que en el mundo se han llevado a cabo políticas para reducir el uso de vehículos particulares en los centros urbanos, las distancias de los viajes han aumentado en la medida que las ciudades se expanden y, a su vez, el deseo de una vida de suburbio, con baja densidad y dependiente del automóvil, se ha vuelto dominante (Banister, 2011).

En la literatura relacionada a la planificación urbana se ha estudiado ampliamente la relación entre la forma urbana y la movilidad. Se ha planteado si es posible tener un impacto en el modo en que nos movemos por medio del entorno construido y la estructura de la ciudad. Varios autores plantean cuáles son las variables de la forma urbana que impactan en la movilidad, y qué se puede hacer a través del diseño para fomentar una movilidad sustentable. También se ha evaluado la importancia de tener en cuenta los factores actitudinales y cómo, si es posible, redireccionar los comportamientos de los ciudadanos hacia tendencias sustentables.

Por medio del estudio del caso de Montevideo podemos ejemplificar la relación de las características físicas de la ciudad con la movilidad. A su vez, podemos analizar los objetivos y estrategias que plantean los planes desarrollados por el gobierno municipal con respecto al desarrollo urbano y movilidad.

\section{MOVILIDAD Y ESTRUCTURA URBANA}

La temática sobre cómo la estructura urbana impacta en la movilidad ha sido discutida ampliamente en la literatura, y es probablemente uno de los temas más estudiados en planificación urbana (Ewing y Cervero, 2009). En un estudio realizado en 1997, en donde resumen varios de los hallazgos de la literatura relacionada a este tema, Cervero y Kockelman definen tres características de la estructura urbana que tienen un impacto en la movilidad: densidad, diversidad y diseño (Cervero y Kockelman, 1997).

\section{Densidad}

Los autores Newman y Kenworthy han realizado una de las investigaciones más extensas y más frecuentemente citadas sobre cómo la densidad impacta en la movilidad (Dieleman et. al., 2001). En su estudio sobre transporte y forma urbana en 32 ciudades del mundo, 


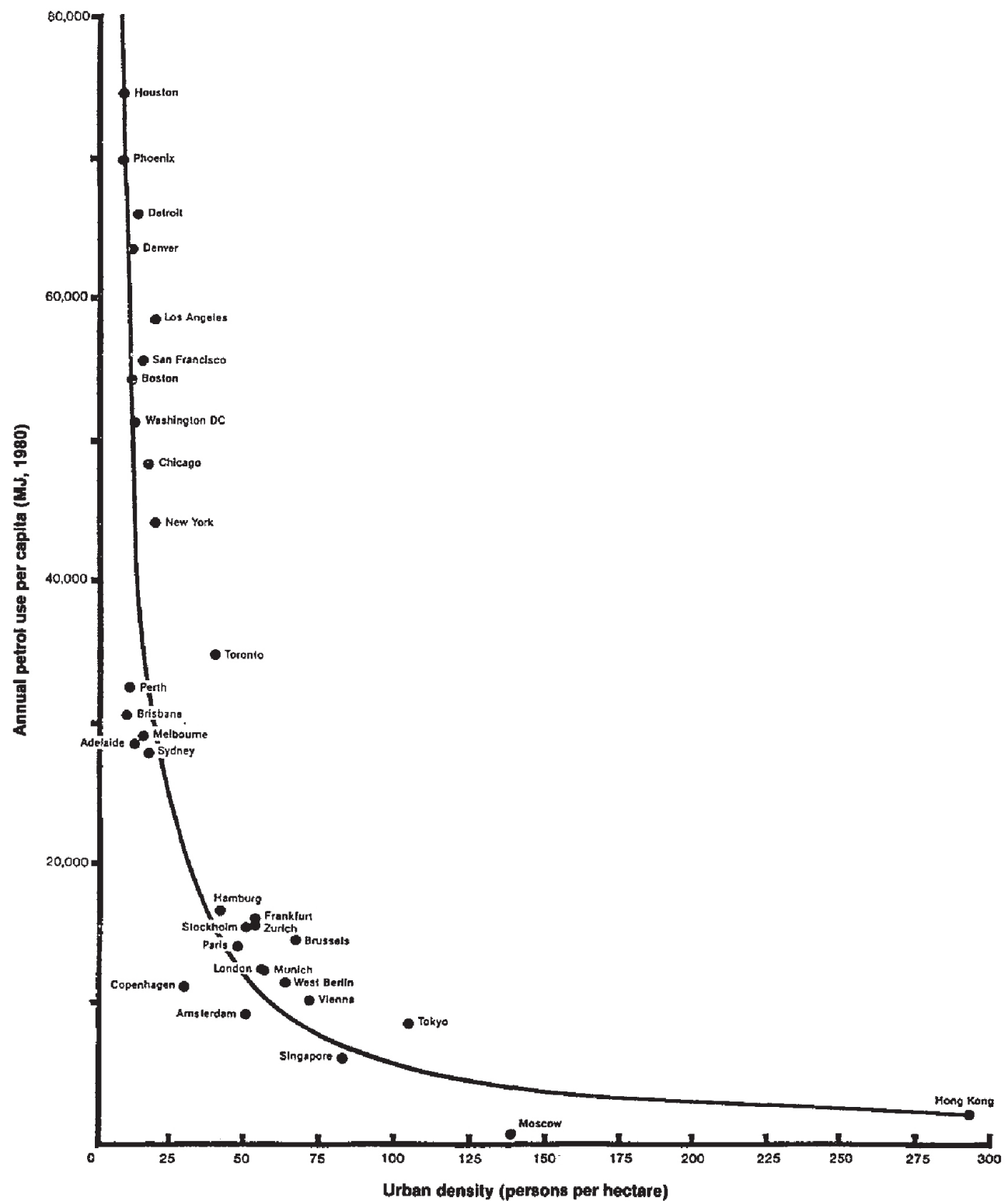

Figura 01.

Uso de combustible per cápita (MJ) vs densidad de población (personas/hectárea) (Newman y Kenworthy, 1991). 
Newman y Kenworthy (1991) sostienen que la densidad de población es la característica más importante en impactar en la movilidad. En esta investigación, comparan por un lado la densidad de habitantes y por otro, el consumo de combustible de vehículos por persona, y demuestran la conexión aparentemente lineal entre ambas variables. En la gráfica que los autores desarrollan a partir de estas variables, se muestra que las ciudades norteamericanas de Houston y Phoenix tienen la menor densidad de población (menos de 25 personas por hectárea) y, en contrapartida, el mayor nivel de consumo de combustible per cápita. El caso opuesto es el de Hong Kong que, con una densidad de casi 300 personas por hectárea, tiene el menor consumo de combustible por persona.

\section{Diversidad}

Según Cervero y Kockelman (1997), la diversidad mide la cantidad de usos del suelo para un área determinada. Bajos niveles de diversidad indican entornos con un solo uso del suelo y, por el contrario, altos niveles de diversidad indican usos del suelo variados.

Newman y Kenworthy consideran también el factor de la diversidad en sus estudios. Los autores establecen que para obtener una movilidad más eficiente es imperioso reducir viajes, y para ello, es necesario que los patrones de densidad de empleo sean similares a los de densidad de población (Newman y Kenworthy, 1999). Es decir que, un tejido urbano diverso en cuanto a sus usos genera que la utilización del automóvil privado se reduzca, ya que las distancias a los distintos destinos son más cortas y eso favorece a peatones y ciclistas.

En esta misma línea escribe Rueda (2002), quien analiza los modelos antagónicos de la ciudad compacta y compleja en contraposición con la ciudad dispersa y difusa. El autor comenta que la tendencia actual de generar ciudad consiste en la implantación de usos y funciones en el territorio de modo disperso y segregado, es decir, con baja diversidad. Desde el punto de vista de la movilidad, esto lleva a que la conexión entre estos usos sólo sea posible con medios mecánicos (red de carreteras), y que, a su vez, el crecimiento de la ciudad sea posible solamente mediante el crecimiento de esta red vial que se transforma entonces en el estructurador del territorio. Este queda así compartimentado, lo que a su vez tiene un impacto en la segregación de la población. Según Rueda, el resultado de este modelo de ciudad dispersa y con baja diversidad es un uso masivo de los medios de locomoción (en general privados), por ende, redes viales saturadas y congestionadas (Rueda, 2002).

\section{Diseño}

Según el estudio de Cervero y Kockelman (1997), el diseño considera las características del sistema de viario en un área determinada. El viario puede variar desde tramas densas con alta conectividad y permeabilidad, a sistemas suburbanos dispersos con baja conectividad (por ejemplo, con cul-de-sacs). Las características que influyen son el tamaño 
promedio de las cuadras, y la cantidad de intersecciones en un área determinada, el ancho de las veredas, ancho de calles, cantidad de cruces peatonales, y otras variables físicas que diferencian entornos orientados al peatón y ciclistas de entornos orientados a vehículos particulares.

Las Naciones Unidas han desarrollado un sistema para evaluar la prosperidad de las ciudades (City Prosperity Index), por medio del cual se miden factores como la productividad, la calidad de vida, las infraestructuras y la sostenibilidad medioambiental de las ciudades. El informe establece que aquellas ciudades con mayor índice de prosperidad, son a su vez aquellas que cuentan con una alta conectividad en su trama de calles. A su vez, el diseño de calles que promueven a peatones y ciclistas, genera un impacto positivo en la calidad de vida y en la inclusión social, y por ende un aumento en el índice de prosperidad de dicha ciudad (UN, 2013).

Por otro lado, la gran brecha que existe entre la conectividad del sistema vial en los centros urbanos y las periferias es un reflejo de las enormes desigualdades en la mayoría de las ciudades del mundo en desarrollo (UN, 2013). La baja conectividad, especialmente en suburbios, periferias y asentamientos informales, genera un gran impacto negativo en la capacidad que tiene la ciudad de proveer las infraestructuras y servicios adecuados a estos sectores del territorio. Una trama de calles bien conectada tiene una gran cantidad de intersecciones, y pocos cul-de-sacs. Al aumentar la conectividad, las distancias de viaje disminuyen, y las opciones tanto de recorridos a hacer como modos de viaje (transporte público, bicicleta, caminata) aumentan, creando un sistema en general más accesible (UN, 2013).

\section{La cuestión de la elección}

A la hora de estudiar cómo la forma urbana y su planificación pueden impactar en la movilidad, algunos autores plantean la necesidad de tener en cuenta otros factores, como son los elementos actitudinales y el comportamiento de los ciudadanos. Las personas deciden sobre cómo moverse por la ciudad y dónde vivir, y estas decisiones no siempre están relacionadas con el entorno construido sino también a elementos culturales, hábitos y aspiraciones.

Guglielmetti et. al. (2017) sostienen que es necesario que los gobiernos municipales ejecuten campañas de gestión de movilidad que ayuden a cambiar hábitos y normas sociales, y aseguran que esto debe ser una parte esencial de los planes estratégicos de movilidad y transporte. Hiselius y Rosqvist (2015) plantean que estas campañas pueden ser la herramienta que unifique los esfuerzos individuales de los ciudadanos, de forma tal de lograr un cambio más contundente y holístico.

Los modos de transporte que las personas elijen también están relacionados con la elección de lugares donde residen. Con respecto a 
esto, Bohte et. al. (2009) señalan que ignorar este factor a la hora de evaluar la movilidad sería sobreestimar el impacto que tiene el entorno construido. Los autores consideran que es importante identificar los hábitos de las personas y fomentar comportamientos sustentables en hogares. Pero, si las personas consideran que el uso del vehículo particular es la mejor opción para moverse, los autores cuestionan si invertir en transporte público puede llegar a cumplir con los objetivos deseados de movilidad sustentable.

Para Dieleman et. al. (2001), la preferencia de los consumidores en cuanto a la elección de lugares donde vivir ha tendido hacia entornos residenciales menos compactos $y$, junto con esto, al alto uso de los vehículos particulares. Según los autores, la relación entre la forma urbana y la elección del modo de transporte es difícil de dilucidar, dado que muchos factores influyen en esta relación. Por ejemplo, las características de cada hogar con respecto a ingreso, composición, actividad laboral, influyen en los comportamientos y elecciones en cuanto cómo eligen moverse. A su vez también influye el propósito del viaje (trabajo, compras para el hogar, etc.).

El factor actitudinal y las características de los hogares agregan una capa de complejidad a la discusión sobre la movilidad, y parecen tan relevantes como las características físicas de la ciudad. La dificultad que presenta esta variable es tener que considerar hábitos y actitudes no necesariamente racionales, que no sólo pueden ser complejas de predecir y medir, sino también de modificar.

\section{EL CASO DE MONTEVIDEO}

Al igual que la mayoría de las ciudades latinoamericanas, Montevideo es sujeto afectaciones tales como una movilidad ineficiente, segregación, polución y altos índices de accidentes de tránsito (CAF, 2011). Utilizando las tres variables definidas por Cervero y Kockelman (1997), podemos analizar la estructura urbana de Montevideo y su relación con la movilidad.

A pesar de que su población se ha mantenido estable por décadas, Montevideo se ha convertido en una ciudad más dispersa (IM, 2010) y, tal como ha sido la tendencia global, su centro ha sufrido un proceso de pérdida de residentes. La migración de personas del centro de la ciudad se ha dado por diferentes motivos, principalmente económicos (IM, 2010), y se ha manifestado básicamente a través de dos fenómenos. El primero y más significativo es el aumento de asentamientos informales en las periferias de la ciudad (Portillo, 2010). E segundo es la migración de las clases mediaalta y alta a los suburbios, concentrados principalmente en la costa este del Área Metropolitana de Montevideo (AMM) (IM, 2013).

Con respecto a la densidad de población, en general la ciudad tiene una densidad 


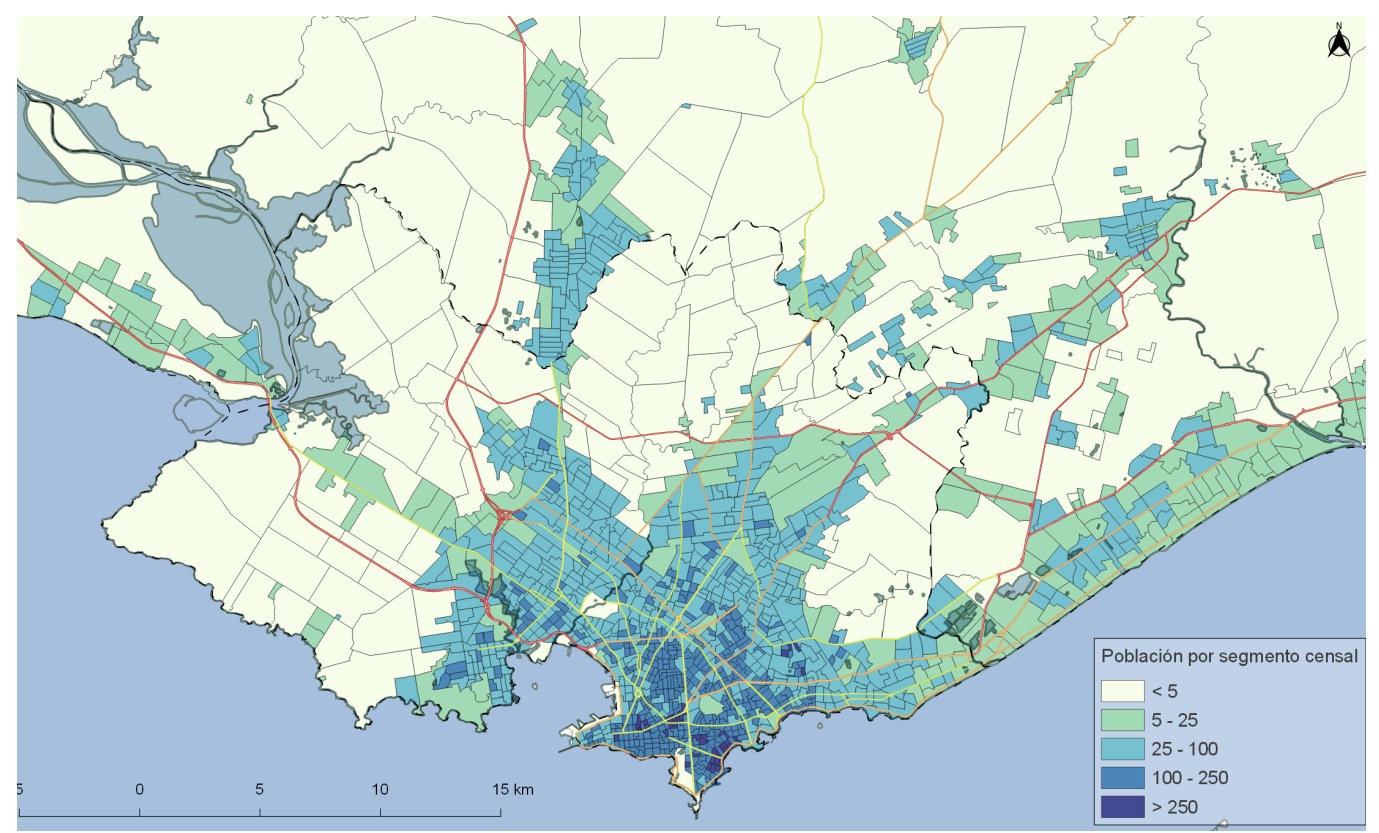

Figura 02.

Densidad (personas/hectárea) en Área Metropolitana Montevideo. Según datos Censo 2011.

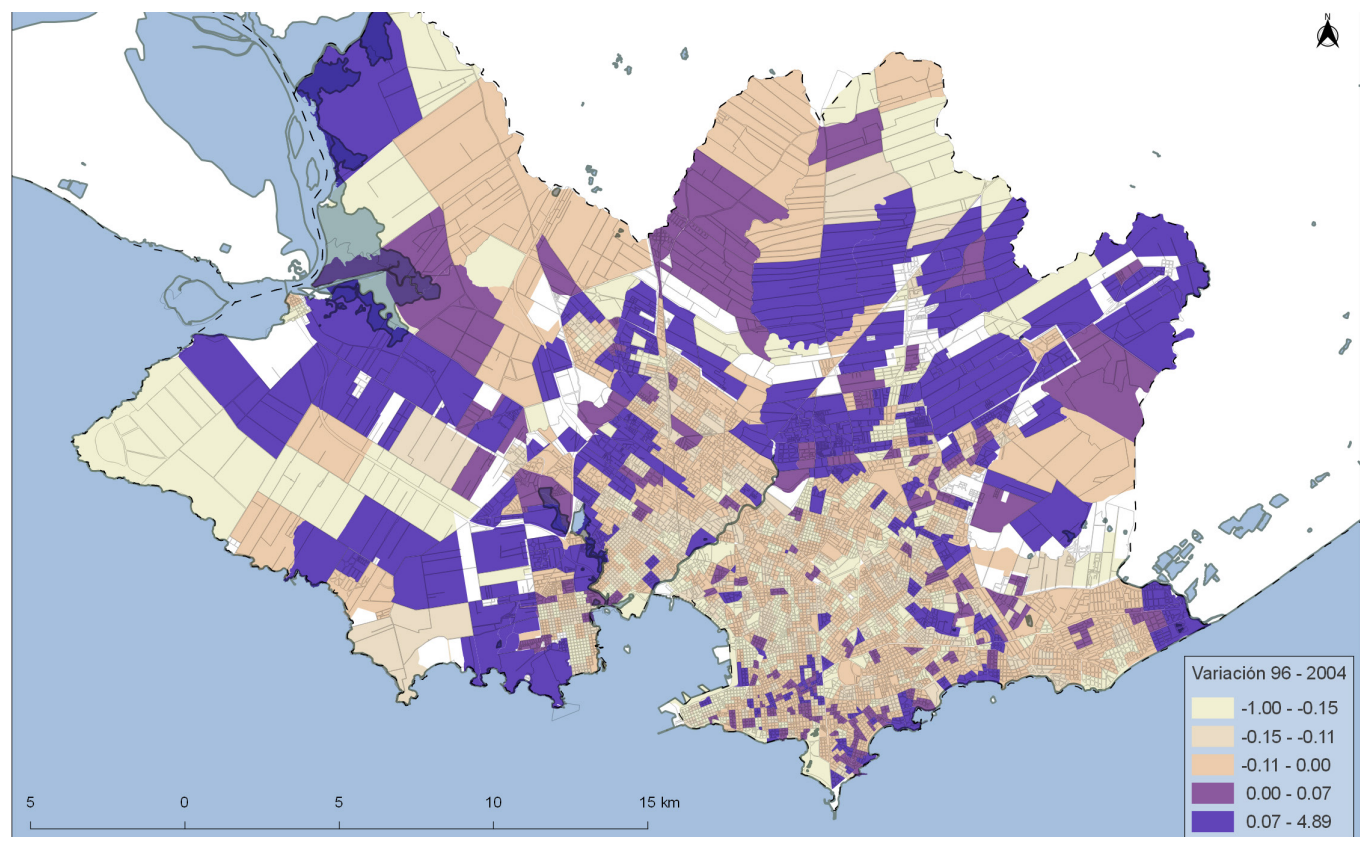

Figura 03.

Variación de población entre 1996 y 2004 por segmento censal en porcentajes. 
relativamente baja, especialmente en sus zonas intermedias y periféricas. Las densidades más altas se concentran en su zona central, en barrios como Centro, Cordón y Pocitos. Desde 1985 las zonas centrales e intermedias de la ciudad han perdido población, mientras que el crecimiento poblacional se ha concentrado en las periferias de Montevideo. La pérdida de población no ha significado pérdida de hogares, ya que estos han crecido debido a su transformación en hogares más pequeños. En el período censal entre 1996 y 2004 es donde es más notorio la polarización entre el aumento de población en las periferias y el descenso en las áreas centrales e intermedias (Bervejillo, 2016).

Si seguimos la línea de razonamiento que establecen Newman y Kenworthy, el fenómeno de disminución de densidad de población podría llevar al aumento de las distancias de viaje (en el AMM pueden ser de más de $30 \mathrm{~km}$ ) y el aumento del uso del vehículo privado como medio de transporte (por medio de aquellos que pueden costearlo), generando congestión y un empeoramiento de la calidad de vida de los ciudadanos en general.

Con respecto a la diversidad, podemos analizar la relación entre actividad laboral y residencial en Montevideo. Tradicionalmente, la oferta laboral en Montevideo estaba concentrada en las áreas centrales, tanto en su centro histórico (Ciudad Vieja), como los barrios Centro y Cordón. En los últimos años, han surgido nuevos emprendimientos de oficinas en otros puntos de la ciudad, principalmente siguiendo la línea de la costa hacia el este (barrios como Punta Carretas y Pocitos). A grandes rasgos, se podría decir que tanto el Centro como Pocitos, además de concentrar la mayor cantidad de oferta de trabajo de la ciudad, son a su vez las zonas de mayor densidad de población de Montevideo, lo que da a priori una relación entre poblacióntrabajo equilibrada y, por ende, barrios con alta diversidad.

Según la encuesta de Movilidad del Área Metropolitana de Montevideo (Mauttone y Hernández, 2017), el Municipio B (que incluye barrios como Ciudad Vieja, Centro, Cordón, y Parque Rodó, entre otros), es la zona que cuenta con mayor atracción de viajes en hora pico de la mañana, seguido por los municipios $\mathrm{C}$ y $\mathrm{CH}$ (que incluye Punta Carretas y Pocitos). A su vez, el Municipio B presenta el mayor valor de producciones de viajes en el horario pico de la tarde. También la encuesta detalla que, con respecto a los viajes con motivo de trabajo, el Municipio B es nuevamente el sector de la ciudad que tiene la mayor cantidad de producción y atracción de viajes. Cerca de la mitad de los viajes producidos y atraídos por el Municipio B se dan intra-municipio, es decir, se generan y tienen como destino algún punto del propio municipio.

La encuesta da una imagen poco detallada de los orígenes y destinos de los viajes en general, ya que considera a los municipios 
como el área mínima de estudio, y estos tienden a ser sectores amplios de territorio y carentes de homogeneidad en cuanto a densidad de población, trabajo, y actividad en general. Además, la cantidad total de viajes atraídos y producidos por el Municipio B no está desagregada por modo, por lo que no se puede determinar si debido a la alta diversidad que cuenta este sector de la ciudad se producen más viajes en modos activos que en vehículos particulares. Del resto de los viajes producidos y atraídos por el municipio B, un gran porcentaje tienen como origen y destino otros municipios y puntos del AMM. Tampoco se aclara en qué modalidad se realizan estos viajes, por lo que es difícil sacar conclusiones.

De todas formas, indistintamente de la modalidad, y por un tema meramente de forma de semicírculo de la ciudad, se produce un efecto de embudo hacia las zonas centrales por vías de acceso puntuales, que genera congestión en las horas pico. A su vez, la encuesta demuestra que en el período entre 2009 y 2016, ha habido un aumento del uso de los vehículos motorizados privados como modo principal de viaje (de $45,4 \%$ a $51,6 \%$, excluyendo los viajes cortos a pie), y a su vez, una disminución del uso de transporte público (de 39,1\% a 35,7\%). Todo esto sumado a que, en general, se ha dado un incremento de las personas que se movilizan en la ciudad, y también un incremento de los viajes por persona (Mauttone y Hernández, 2017), puede explicar la congestión en zonas centrales.
Este efecto de embudo parece solo ser salvable en la medida en que otros puntos del territorio empiecen a tener una relación residenciatrabajo más equilibrada, especialmente las zonas intermedias que ya cuentan con una infraestructura apropiada para alojar tanto residentes como trabajadores. De esta forma, se generarían viajes más cortos y por ende se fomentarían los modos de viaje sustentables. Sería interesante que la Encuesta de Movilidad contara con un análisis más detallado del territorio, por medio de unidades de análisis más pequeñas, para determinar exactamente cuáles son los orígenes y destinos de los viajes dentro de los municipios y contrastarlo con la relación de densidad de población y trabajadores de cada sector en particular.

Con respecto al diseño de la trama urbana, la ciudad de Montevideo en general cuenta con una alta conectividad, brindada por una grilla de calles que caracteriza grandes partes de la ciudad. Sin embargo, la situación en las periferias del área metropolitana es distinta. Aquí, el crecimiento se ha dado en pequeños fraccionamientos adheridos a las grandes vías de acceso a la ciudad. Estos fraccionamientos, a pesar de que en sí mismos son retículas, son independientes y no se conectan entre sí, por lo que dependen de las rutas como elemento unificador. Esta situación se agrava en los asentamientos informales, cuyos sistemas intrincados de pasajes y calles hacen muy difícil la llegada de servicios y la accesibilidad en 


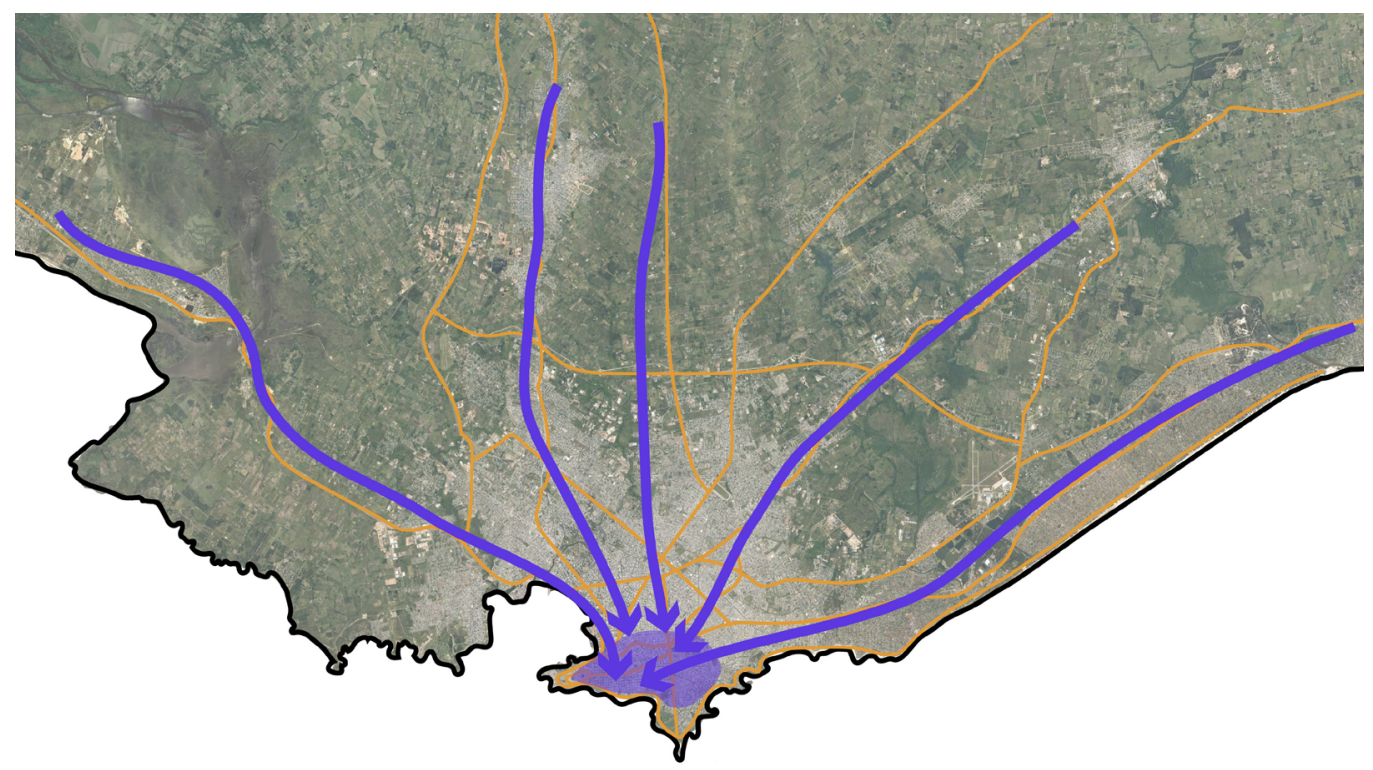

Figura 04.

Esquema estructura vial Montevideo.

general.

Desde hace unos años ha surgido en el AMM, al igual que en muchas otras ciudades latinoamericanas, el fenómeno de los barrios privados. La Intendencia de Canelones permite la construcción de barrios privados, que normalmente cuentan con un diseño poco permeable, en un sector específico del departamento (Camino de los Horneros). Estos emprendimientos han creado suburbios que sólo pueden accederse en vehículos particulares y que están totalmente desconectados del resto de la trama urbana.

\section{La elección de los montevideanos}

Los usuarios del sistema de transporte público en Montevideo han bajado, al mismo tiempo que el uso del auto y la cantidad total de personas que se mueven es mayor (Mauttone y Hernández, 2017). Parte de esto puede explicarse por una mejora en la economía del país luego de la crisis económica del 2002, que ha llevado a un mayor poder adquisitivo y a su vez a una baja de los precios de los automóviles y motos (CAF, 2011). Uno de los aspectos peor evaluados por los montevideanos con respecto al sistema de transporte público capitalino es el costo del mismo (Mauttone y Hernández, 2017). Es posible que algunos usuarios opten por comprar un vehículo particular (especialmente motos) dado que, dependiendo de los viajes que necesiten hacer, es factible que sea una opción más económica que el transporte colectivo. 


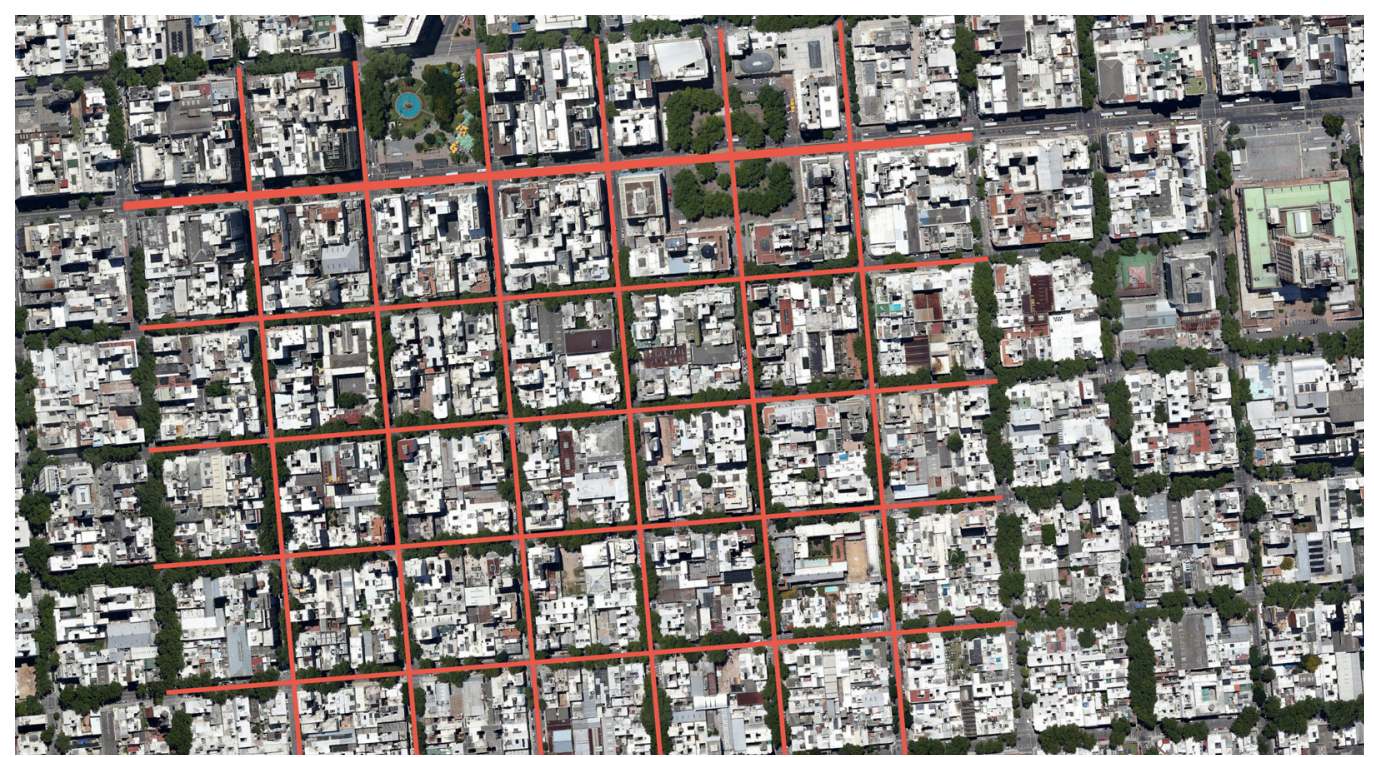

Figura 05.

Trama de calles en área céntraica de Montevideo. La grilla, que cuenta con un alto índice de cruces, le otorga gran conectividad y permeabilidad al sistema.

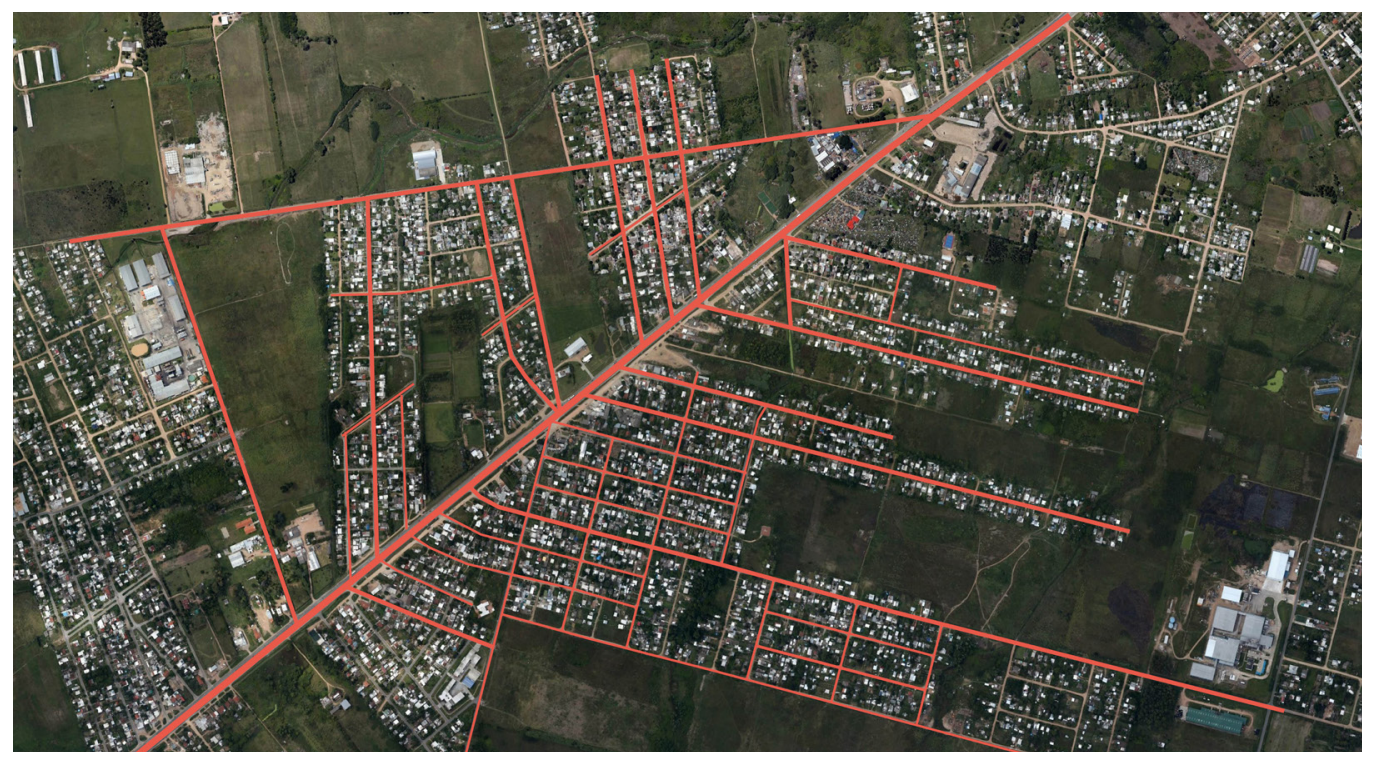

Figura 06.

Trama de calles en periferia del Área Metropolitana de Montevideo (Barros Blancos, Canelones). La ruta es el principal estructurador del sistema, a partir del cual se organizan los tejidos, que tienen poca conectividad entre sí. 


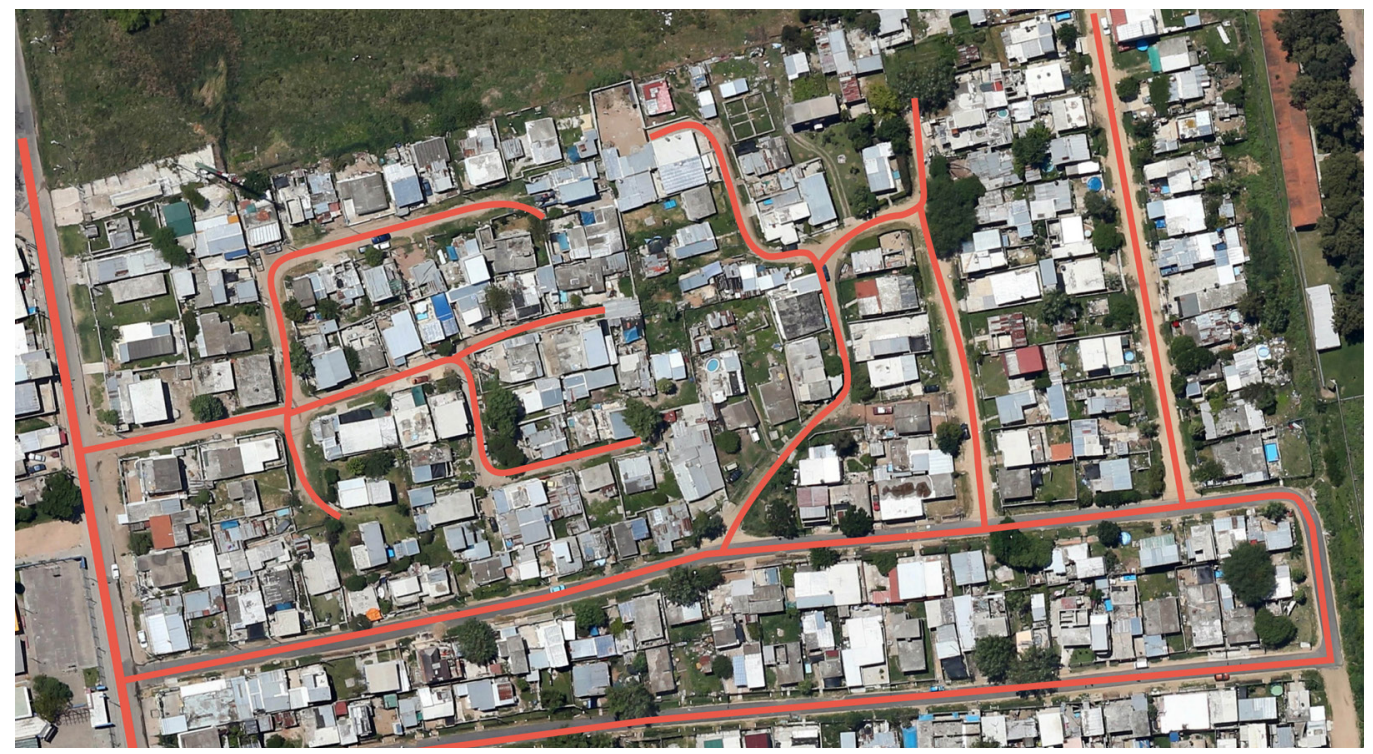

Figura 07.

Trama de calles en asentamiento informal en Montevideo (Santa Catalina). Sistema intrincando de pasajes y calles que no se conectan entre sí, lo que complejiza la accesibilidad.

Otro motivo que puede influir en la decisión de los montevideanos de utilizar vehículos propios es el tiempo de traslado. La velocidad promedio del transporte público en Montevideo es de 16 $\mathrm{km} / \mathrm{hr}$, y sólo 6 a $8 \mathrm{~km} / \mathrm{hr}$ en las zonas centrales (IM, 2010), lo que lleva a que esta modalidad tenga los tiempos de viajes más largos. Esto, sumado al aspecto aspiracional sobre la tenencia de un vehículo particular, hace del transporte público un modo poco deseable o rentable, y las personas optan por otros modos en la medida de sus posibilidades.

Mientras que en el mundo la vida urbana está “de moda" y estilos de vida sustentables están siendo adoptados por muchas personas (Banister, 2011), las encuestas demuestran lo contrario para Montevideo. A pesar de que se ha impulsado la construcción de bicisendas en zonas centrales, ésta ha sido lenta y poco regulada. Más allá de algunos movimientos de la sociedad civil que promueven la movilidad sustentable, no se está dando un cambio de hábitos lo suficientemente contundente para contrarrestar la tendencia del uso del vehículo particular.

\section{Plan de Movilidad de Montevideo}

En 2010 la Intendencia de Montevideo presenta el Plan de Movilidad, cuyo objetivo es “lograr un transporte más eficiente, cómodo, económico, ambientalmente sustentable y fundamentalmente democrático" (IM, 2010, 
pág.7). A pesar de que actualmente su vigencia está cuestionada, especialmente por el resultado negativo obtenido en la implementación de las primeras fases del plan, al momento la Intendencia no ha publicado una revisión del documento.

El plan reconoce que los cambios que ha sufrido la ciudad en las últimas décadas están llevando a una crisis de la movilidad, aunque, según se detalla, una crisis no totalmente instalada. En respuesta a esta crisis se plantea una movilidad urbana basada en términos de equidad social y sustentabilidad ambiental, por ende, se priorizan las acciones relacionadas al transporte colectivo y a los modos activos de viaje. El Plan de Movilidad toma como punto de partida en Plan de Ordenamiento Territorial de 1998 (Plan Montevideo). Según se detalla, ambos planes comparten una visión estratégica y se recogen del Plan Montevideo las directrices generales referidas a vialidad y transporte.

En general, el plan promueve una movilidad sustentable y un vínculo con el desarrollo urbano, pero carece de detalle sobre cómo encarar problemáticas como la migración de población hacia las periferias y temas actitudinales sobre la elección de modos de viaje. Se detalla que "se hará un esfuerzo por integrar la planificación del transporte con la planificación del crecimiento urbano, los usos del suelo y las antiguas y nuevas centralidades" (IM, 2010, pág. 23), pero no se especifica cómo esto se va a llevar a cabo. Se proponen corredores de transporte y ubicación de terminales "en el límite de las áreas urbanas periféricas buscando contribuir a consolidar los tejidos urbanos, reforzando centralidades existentes o constituyéndose como generadoras de nuevas centralidades" (ídem, pág.46), pero no hay un detalle de una normativa urbana que acompañe estas infraestructuras viales.

La Encuesta de Movilidad de Montevideo y los estudios realizados por el Observatorio de Movilidad han demostrado una tendencia al aumento del parque automotor y una baja en los usos de modos de viaje sustentables (Mauttone y Hernández, 2017; Vasconcellos y Mendonça, 2016). Ocho años luego de su publicación, el Plan de Movilidad no ha logrado revertir esta tendencia, por lo que resulta evidente la necesidad de una revisión del mismo.

\section{Directrices Departamentales}

En 2012, la Intendencia de Montevideo publica las Directrices Departamentales de Ordenamiento Territorial y Desarrollo Sostenible (DDOTDS). Las DDOTDS surgen como parte del proceso de revisión del Plan Montevideo de 1998, "conformando el instrumento que contendrá las decisiones estructurales y estratégicas sobre el ordenamiento territorial del territorio" (IM, 2012, pág. 4). Uno de los aspectos a lo que refieren las DDOTDS es a la formulación de orientaciones generales para las políticas sectoriales (incluyendo movilidad).

El discurso de las DDOTDS está acorde con la 


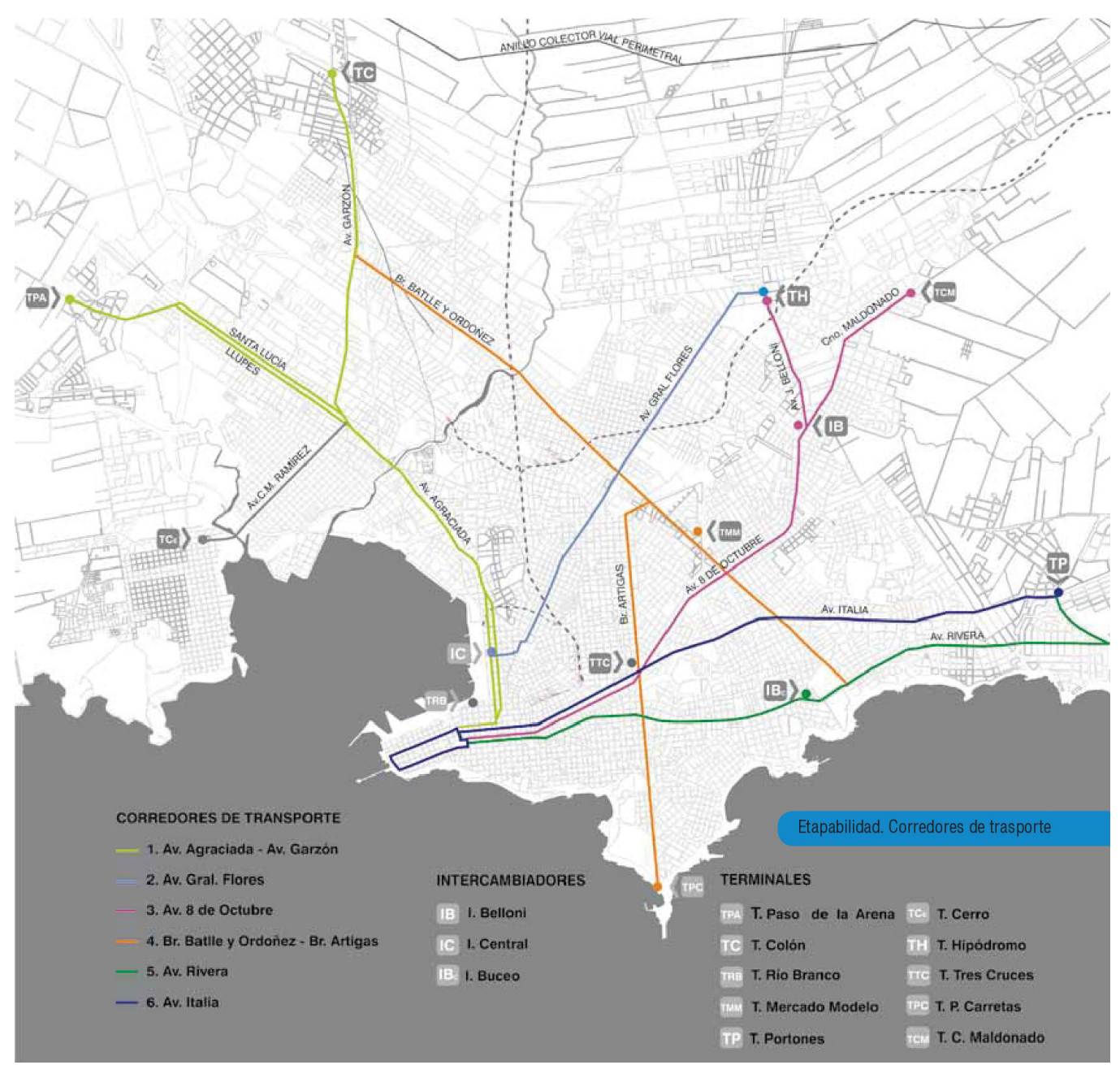

Figura 08.

Corredores de transporte, intercambiadores y terminales según el Plan Movilidad. Se plantean como corredores las siguientes calles: Av. Agraciada, Av. Garzón, Av. Carlos María Ramírez, Bvar. Batlle y Ordónez, Bvar. Artigas, Av. 8 de Octubre, Av. Gral. Flores, Av. Italia, Av. Rivera. (IM, 2010) 


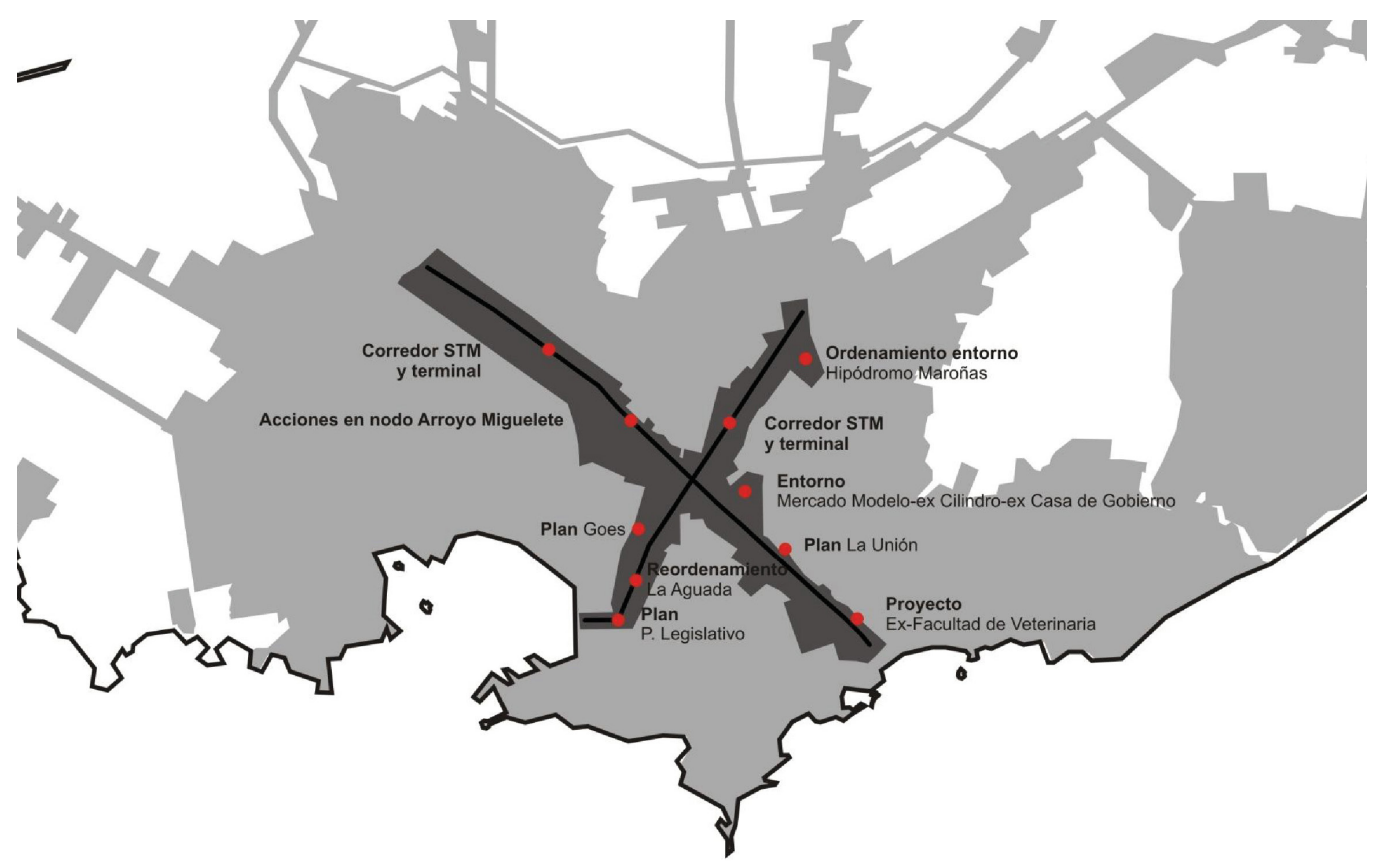

Figura 09.

Territorios estratégicos según DDOTDS: Ejes urbanos (Bulevar Batlle y Ordóñez y Av. General Flores). (IM, 2012)

literatura vista anteriormente. Sus lineamientos van en la línea de compactar, redensificar, diversificar y generar un diseño accesible e inclusivo. A su vez, se reconoce la importancia del desarrollo vial y urbano en conjunto. Hay una intención clara de integrar la planificación de la movilidad con la planificación integral del territorio adyacente a los corredores y terminales. Se entiende esencial la conectividad vial que reequilibre la estructura concéntrica actual de Montevideo, habilitando nuevas vinculaciones.

El documento plantea una serie de territorios estratégicos, dentro de los cuales incluye dos ejes urbanos (Bvar. Batlle y Ordóñez y Av. Luis Alberto de Herrera, y Av. Gral. Flores), y propone una serie de intervenciones a realizar en cada uno. Ambos ejes son también propuestos como corredores en el Plan de Movilidad.

Sin embargo, debido a que la naturaleza de este documento es de carácter orientador y general, los puntos que desarrolla son sólo lineamientos generales, y no hay un detalle concreto de cómo llevarlos a cabo más allá de nombrar algunas posibles intervenciones a realizar. Es decir, nuevamente, no hay una normativa específica que de alguna forma refleje estas intenciones. La normativa vigente, entonces, sigue siendo 

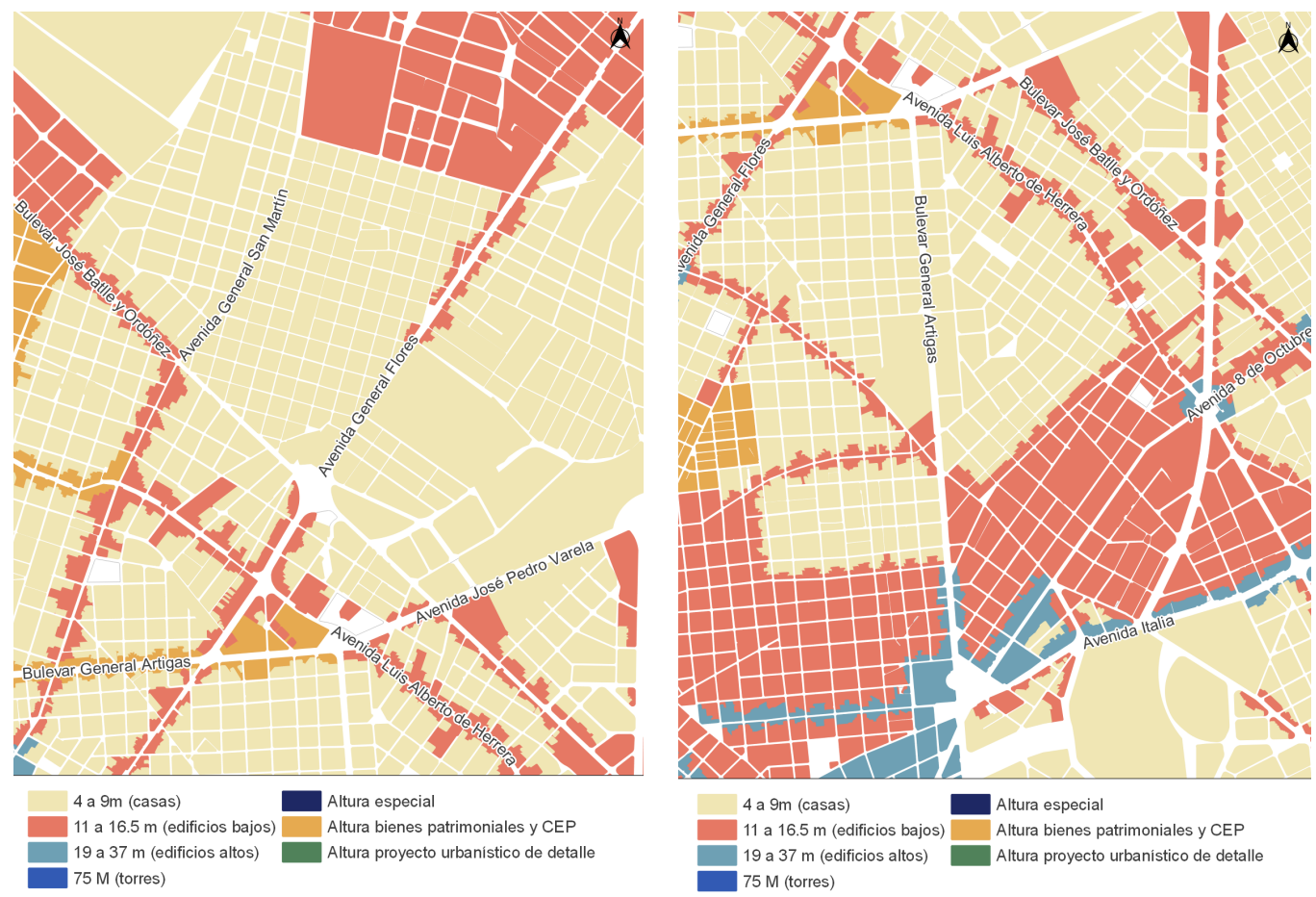

Figura 10 y 11.

Rangos normativos de altura de la edificación en Montevideo, según Plan Montevideo (1998). Sección. Sector Bvar. Batlle y Ordóñez y Av. Gral. Flores. (Bervejillo, 2016).

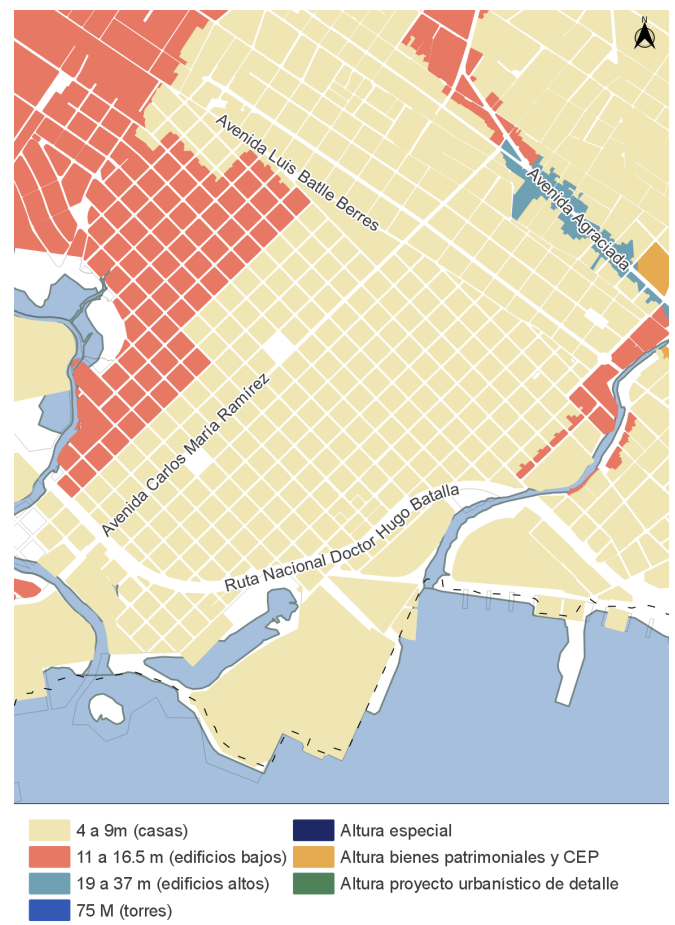

Figura 12.

Rangos normativos de altura de la edificación en Montevideo, según Plan Montevideo (1998). Sección. Sector Av. Carlos María Ramírez. (Bervejillo, 2016). 
la planteada en el Plan Montevideo en el año 1998. Con respecto a las alturas máximas permitidas, si bien se proponen alturas hasta $37 \mathrm{~m}$ en zonas céntricas y en algunos ejes principales, en general el plan propone alturas bajas (máximo 9m), especialmente en las zonas intermedias, y a su vez varios ejes no están jerarquizados. La altura promedio de $9 \mathrm{~m}$ en las áreas intermedias no es una normativa que estimule la densificación y, a pesar de que en teoría permitiría una densificación moderada, no convierte a estas zonas en atractivas para la inversión (Bervejillo, 2016).

De los corredores viales propuestos por el Plan de Movilidad, sólo dos son retomados en las DDOTDS como territorios estratégicos (Av. Gral. Flores y Bvar. Batlle y Ordoñez). El resto de los corredores no son destacados, por lo que no parece haber una coordinación total entre ambos planes a la hora de definir qué ejes son estratégicos. En el Plan Montevideo, tanto Av. Gral Flores como Bvar. Battle y Ordóñez cuentan en grandes tramos con una altura máxima permitida de 16,5 metros, lo que permitiría la construcción de edificios bajos de hasta 6 plantas, pero justamente en el punto donde los dos ejes se cruzan, la altura permitida es más baja, de 9 metros (salvo en el tramo de Av. Gral. Flores el sur). La normativa entonces no parece estar acorde a la jerarquización que se le pretende dar a estos corredores.

Otros de los corredores propuestos por el Plan de Movilidad es Bvar. Artigas (tramo nortesur). Este eje cuenta con una altura máxima permitida también de 9 metros desde Tres Cruces hasta el encuentro con Av. Luis Alberto de Herrera. Sin embargo, se permite una altura mayor de 16,5 metros de Bvar. Artigas al este, dentro de la trama del barrio La Blanqueada. Algo similar sucede en el barrio La Teja, donde en la Av. Carlos María Ramírez (otro de los corredores planteados por el Plan Movilidad), la altura propuesta es de 9 metros, mientras que el sector noroeste del barrio (hacia el arroyo Pantanoso) cuenta con una normativa de 16,5 metros. No queda claro cuál es el criterio con el cual esta normativa fue determinada, pero no parece haber, al menos en estos puntos, una intención de jerarquizar los corredores por sobre el resto de la trama.

\section{COMENTARIOS FINALES}

Montevideo está experimentando, como muchas otras ciudades en el mundo, un proceso de dispersión territorial que va de la mano con un aumento del parque automotor. Esto ha provocado no sólo un incremento de la congestión, polución, accidentes de tránsito, sino también ha colaborado en acentuar la problemática de la segregación social.

La Encuesta de Movilidad realizada por la Intendencia de Montevideo es un instrumento esencial para poder hacer una planificación detallada. Sería importante que se realizara este análisis en unidades de territorio más pequeñas y homogéneas, de forma tal de poder comprender exactamente cómo se 
mueven los montevideanos con respecto a las características físicas de los entornos donde residen y trabajan.

En su discurso, tanto las Directrices Departamentales como el Plan de Movilidad apuntan a un desarrollo urbano sustentable, pero en los hechos no plantean normativas concretas y en conjunto que unifiquen el desarrollo urbano y la planificación de la movilidad. Sería relevante, entonces, que se revisara la normativa actual (principalmente en cuanto a las alturas permitidas) y que, en coordinación con el Plan de Movilidad, se promoviera la densificación en los ejes estratégicos. A su vez, deberían incluirse campañas de gestión de la movilidad contundentes, que apunten a lograr un cambio en el comportamiento de los ciudadanos. Si las personas continúan migrando hacia las periferias, y si no utilizan el transporte público porque no es una opción económicamente viable o deseable, el sistema de movilidad sustentable planteado no va a ser exitoso.

Según Banister (2011), en el mundo hay muchos ejemplos de reducciones de uso de vehículos particulares en centros urbanos, principalmente a través de métodos de control de la demanda (aumentando tarifas de estacionamiento o de acceso a determinados puntos de la ciudad), y también a través de la inversión en sistemas de transporte público, en infraestructuras apropiadas para peatones y ciclistas, y algunas medidas para intentar evitar el uso de vehículos privados por solo una persona (car-sharing). A su vez también a partir de la planificación urbana, se han creado barrios que incluyen emprendimientos de usos mixtos, y se han fomentado los emprendimientos alrededor de nodos de transporte público (transport oriented developments). En todos los casos, la intención es reducir la necesidad de viajar y reducir las distancias de viaje, lo que fomenta el uso del transporte público y los modos activos. La clave está en proveer entornos de calidad, con fácil acceso a servicios, de forma tal que las personas no tengan que viajar grandes distancias en la ciudad (Banister, 2011).

La Ley de Vivienda Promovida del año 2011, impulsada por la Agencia Nacional de Vivienda (MVOTMA), puede jugar un rol importante en la redensificación de las zonas centrales e intermedias de Montevideo, ya que promueve la construcción y refacción de viviendas en sectores consolidados. Sería interesante evaluar cuál ha sido el impacto de esta ley, no sólo en brindar vivienda a los sectores más vulnerables (que es su objetivo primordial), sino también en generar un aumento de la densidad y diversidad en distintos puntos del territorio. 


\section{BIBLIOGRAFÍA}

BANISTER, D. (2008). The sustainable mobility paradigm. Transport Policy, 15, 73-80.

BANISTER, D. (2011). Cities, mobility and climate change. Journal of Transport Geography, (19), 1538-1546.

BERVEJILLO, F. (2016). Informe de desarrollo urbano. Presentado por el Consorcio Arte-lia-Halcrow-Rhama-CSI para el Plan Director de Saneamiento y Drenaje Urbano de Montevideo (IM - División Desarrollo Ambiental - División Saneamiento). Montevideo: IM.

BOHTE, W., MAAT, K., \& VAN WEE, B. (2009). Measuring attitudes in research on residential self-selection and travel behaviour: a review of theories and empirical research. Transport Reviews, 29(3), 325-357.

CAF. (2011). Desarrollo urbano y movilidad en América Latina. Caracas: CAF.

CERVERO, R.; \& KOCKELMAN, K. (1997). Travel demand and the 3Ds: density, diversity, and design. Transportation Research, 2, 199219.

DIELEMAN, F., DIJST, M., \& BURGHOUWT, G. (2002). Urban form and travel behaviour: micro-level household attributes and residential context. Urban Studies, 39(3), 507-527.

EWING, R., \& CERVERO, R. (2010). Travel and the built environment. Journal of the American Planning Association, 7(3), 265-294.

GUGLIELMETTI, R., TONI, M., RAHARJO, H., DI PIETRO, L., \& PETROS, S. (2017). Does the servi- ce quality of urban public transport enhance sustainable mobility? Journal of Cleaner Production, (174), 1566-1587.

HISELIUS, L.W., \& ROSQVIST, L.S. (2015). Mobility management campaigns as part of the transition towards changing social norms on sustainable travel behavior. Journal of Cleaner Production (123), 34-41.

IM (1998). Plan Montevideo. Plan de Ordenamiento Territorial 1998 - 2005. Montevideo: IM.

IM (2010). Plan de Movilidad, hacia un sistema de movilidad accesible, democrático y eficiente 2010-2020. Montevideo: IM.

IM. (2012). Directrices Departamentales de Ordenamiento Territorial y Desarrollo Sostenible de Montevideo. Recuperado de: http://www. montevideo.gub.uy/institucional/politicas/ directrices-departamentales-de-ordenamiento-territorial-y-desarrollo-sostenible-de-montevideo

IM (2013). Informe Censos 2011: Montevideo y Área Metropolitana. Recuperado desde http://www.montevideo.gub.uy/sites/default/ files/informe_censos_2011_mdeo_y_area_ metro.pdf

MAUTTONE, A., \& HERNÁNDEZ, D. (2017). Encuesta de movilidad del área metropolitana de Montevideo. Principales resultados e indicadores (report). Montevideo: CAF, Intendencia de Montevideo, Intendencia de Canelones, Intendencia de San José, Ministerio de Transporte y Obras Públicas, Universidad de 


\section{REFERENCIAS IMÁGENES}

la República, PNUD Uruguay.

NEWMAN, P; \& KENWORTHY, J. (1991). Transport and urban form in thirty-two of the world's principal cities. Transport Reviews: A Transnational Transdisciplinary Journal, 11(3), 249 272.

NEWMAN, P; \& KENWORTHY, J. (1999). Sustainability and cities. Overcoming automobile dependence. Washington D.C.: Island Press.

PORTILLO, A. (2010). Vivienda y sociedad: La situación actual de la vivienda en Uruguay. Recuperado desde http://www.fadu.edu. uy/investigacion/files/2015/07/INVESTIGACION-DE-VIVIENDA-VERSION-ULTIMA-portiIlo-1.pdf

RUEDA, S. (2002). Modelos urbanos y sostenibilidad. En I Congreso de Ingeniería Civil, Territorio y Medio ambiente, Madrid. Recuperado desde http://www.bvsde.paho.org/bvsacd/ cd30/modelos.pdf

UN. (2013). Streets as public spaces and drivers of urban prosperity. Nairobi: UN-Habitat.

UNDP URUGuAY. (2012). Políticas de tiempo, movilidad y transporte público: rasgos básicos, equidad social y de género. Recuperado desde http://www.uy.undp.org/content/ uruguay/es/home/library/poverty/publication_1.html

VASCONCELLOS, E. A., \& MENDONÇA, A. (2016). Observatorio de Movilidad Urbana: Informe 2015-2016. (resumen ejecutivo). Caracas: CAF. Recuperado desde http://scioteca.caf. com/handle/123456789/981
Figura 01. NEWMAN, P; KENWORTHY, J. (1991). Transport and urban form in thirty-two of the world's principal cities. Transport Reviews: A Transnational Transdisciplinary Journal, (11:3), p. 249-272.

Figura 02. Elaboración: Arq. Gonzalo Pastorino. Fuente: INE.

Figura 03. Elaboración: Arq. Gonzalo Pastorino. Fuente: INE.

Figura 04. Elaboración propia. Fuente: Google Earth.

Figura 05. Elaboración propia. Fuente: Google Earth.

Figura 06. Elaboración propia. Fuente: Google Earth.

Figura 07. Elaboración propia. Fuente: Google Earth.

Figura 08. IM (2010). Plan de Movilidad, hacia un sistema de movilidad accesible, democrático y eficiente 2010-2020. Montevideo: IMM.

09. IM. (2012). Directrices Departamentales de Ordenamiento Territorial y Desarrollo Sostenible de Montevideo. Recuperado de: http:// www.montevideo.gub.uy/sites/default/files/ informe_de_elaboracion_y_participacion-noviembre12_0.pdf (10 de mayo 2018).

10. BERVEJILLO, F. (2016). Informe de desarroIlo urbano. Presentado por el Consorcio Artelia-Halcrow-Rhama-CSI para el Plan Director de Saneamiento y Drenaje Urbano de Monte- 
video (IM - División Desarrollo Ambiental - División Saneamiento). Montevideo: IM.

11. BERVEJILLO, F. (2016). Informe de desarroIlo urbano. Presentado por el Consorcio Artelia-Halcrow-Rhama-CSI para el Plan Director de Saneamiento y Drenaje Urbano de Montevideo (IM - División Desarrollo Ambiental - División Saneamiento). Montevideo: IM.

12. Bervejillo, F. (2016). Informe de desarrollo urbano. Presentado por el Consorcio Artelia-Halcrow-Rhama-CSI para el Plan Director de Saneamiento y Drenaje Urbano de Montevideo (IM - División Desarrollo Ambiental - División Saneamiento). Montevideo: IM. 